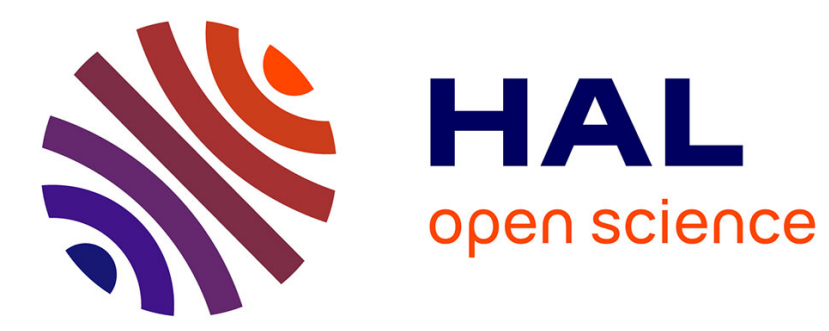

\title{
Shaking Forces Minimization of High-Speed Robots via an Optimal Motion Planning
}

Sébastien Briot, Vigen Arakelian, Nayelli Sauvestre, Jean-Paul Le Baron

\section{To cite this version:}

Sébastien Briot, Vigen Arakelian, Nayelli Sauvestre, Jean-Paul Le Baron. Shaking Forces Minimization of High-Speed Robots via an Optimal Motion Planning. 18th CISM-IFToMM Symposium on Robot Design, Dynamics, and Control (ROMANSY 2010), Jul 2010, Udine, Italy. pp.307-314, 10.1007/978-3-7091-0277-0_36. hal-00460090

\section{HAL Id: hal-00460090 https://hal.science/hal-00460090}

Submitted on 25 Jun 2019

HAL is a multi-disciplinary open access archive for the deposit and dissemination of scientific research documents, whether they are published or not. The documents may come from teaching and research institutions in France or abroad, or from public or private research centers.
L'archive ouverte pluridisciplinaire HAL, est destinée au dépôt et à la diffusion de documents scientifiques de niveau recherche, publiés ou non, émanant des établissements d'enseignement et de recherche français ou étrangers, des laboratoires publics ou privés. 


\title{
Shaking Forces Minimization of High-Speed Robots via an Optimal Motion Planning
}

\author{
S. Briot ${ }^{1}$, V. Arakelian ${ }^{2}$, N. Sauvestre ${ }^{2}$ and J.-P. Le Baron ${ }^{2}$ \\ ${ }^{1}$ Institut de Recherches en Communications et Cybernétique \\ de Nantes (IRCCyN), Nantes, FRANCE \\ Sebastien.Briot@irccyn.ec-nantes.fr \\ ${ }^{2}$ Institut National des Sciences Appliquées (INSA), Rennes, FRANCE \\ vigen.arakelyan@insa-rennes.fr \\ nayelli_sauvestre@hotmail.com \\ jean-paul.le-baron@insa-rennes.fr
}

\begin{abstract}
This paper deals with the problem of shaking force balancing of high-speed robots based on a new optimal trajectory planning approach. The aim of the new approach is the optimal path planning of the robot links centre of masses, which allows a considerable reduction of the variable inertia forces transmitted to the robot frame. The efficiency of the suggested method is illustrated by a numerical simulation of a planar two links $2 R$ serial robot, in which reductions in the shaking force of $63 \%$ and in input torque of $84 \%$ are achieved.
\end{abstract}

\section{Introduction}

A primary objective of linkage balancing is to cancel or reduce the variable dynamic loads transmitted to the frame and surrounding structures. Different approaches and solutions devoted to this problem have been developed and documented for one degree of freedom mechanisms (Lowen et al., 1983), (Arakelian et al., 2000), (Arakelian and Smith, 2005). A new field for their applications is the design of mechanical systems for fast manipulation, which is a topical problem in advanced robotics.

The balancing of a mechanism is generally carried out by two steps: (i) the cancellation (or reduction) of the shaking force and (ii) the cancellation (or reduction) of the shaking moment. Traditionally, the cancellation of the shaking force transmitted to the robot frame can be achieved via adding counterweights in order to keep the total centre of mass of moving links stationary (Lowen et al., 1983), (Arakelian et al., 
2000). However, this approach leads to the increase in the total mass of the mechanical systems and consequently the increase in input torques.

With regard to the shaking moment balancing of robots, the following approaches were developed: (i) balancing by counter-rotations (Berkof, 1973), (Dresig et al., 1994), (Arakelian and Smith, 1999), (Herder and Gosselin, 2004), (ii) balancing by adding four-bar linkages (Gosselin et al., 2004), (Ricard and Gosselin, 2000), (iii) balancing by optimal trajectory planning (Papadopoulos and Abu-Abed, 1994), (Fattah and Agrawal, 2006), (Arakelian and Briot, 2008) and (iv) balancing by adding an inertia flywheel rotating with a prescribed angular velocity (Arakelian and Smith, 2008).

It should be noted that the complete dynamic balancing can only be reached by a considerably complicated design of initial robot mechanisms and by unavoidable increase in the total mass. This is the raison why we focused our research studies on the development of robot balancing methods via optimal motion planning approaches, i.e. without modification of the initial mechanical structure and without any adding masses.

The paper is organized as follows. In the next part, the suggested optimal motion planning is described. Then, for illustration of the efficiency of this approach, simulations carried out using ADAMS software for a planar two links $2 R$ serial robot are presented. Finally, conclusions are drawn in the last section.

\section{Minimization of the Shaking Forces via an Opti- mal Motion Planning of the Total Mass Centre of Moving Links}

The shaking forces $\mathbf{f}^{\text {sh }}$ of a robot can written in the form:

$$
\mathbf{f}^{s h}=m_{t o t} \ddot{\mathbf{x}}_{S}
$$

where $m_{t o t}$ is the total mass of the moving links of a robot and $\ddot{\mathbf{x}}_{S}$ the acceleration of the total mass centre. The classical balancing approach consists in adding counterweights in order to keep the total mass centre of moving links stationary. In this case, $\ddot{\mathbf{x}}_{S}=0$ for any configuration of the mechanical system. But, as a consequence, the total mass of the robot is considerably increased. Thus, in order to avoid this drawback, in the present study, a new approach is proposed, which consists of the optimal control of the total mass centre of moving links. Such an optimal motion planning allows the reduction of the total mass centre acceleration and, consequently, the reduction of the shaking force.

Classically, robot displacements are defined considering either articular coordinates $\mathbf{q}$ or Cartesian variables $\mathbf{x}$. Knowing the initial and final robot configurations at time $t_{0}$ and $t_{f}$, denoted as $\mathbf{q}_{\mathbf{0}}=\mathbf{q}\left(t_{0}\right)$ and $\mathbf{q}_{\mathbf{f}}=\mathbf{q}\left(t_{f}\right)$, or $\mathbf{x}_{\mathbf{0}}=\mathbf{x}\left(t_{0}\right)$ and $\mathbf{x}_{\mathbf{f}}=\mathbf{x}\left(t_{f}\right)$, in the case 
of the control of the Cartesian variables, the classical displacement law may be written in the form:

$$
\mathbf{q}(t)=s_{q}(t)\left(\mathbf{q}_{\mathbf{f}}-\mathbf{q}_{\mathbf{0}}\right)+\mathbf{q}_{\mathbf{0}}
$$

or

$$
\mathbf{x}(t)=s_{x}(t)\left(\mathbf{x}_{\mathbf{f}}-\mathbf{x}_{\mathbf{0}}\right)+\mathbf{x}_{\mathbf{0}}
$$

where $s_{q}(t)$ and $s_{x}(t)$ may be polynomial (of orders 3, 5 and higher), sinusoidal, bang-bang, etc. laws (Khalil and Dombre, 2002).

From expression (1), we can see that the shaking force, in terms of norm, is minimized if the norm $\left\|\ddot{\mathbf{x}}_{S}\right\|$ of the masses centre acceleration is minimized along the trajectory. This means that if the displacement $\mathbf{x}_{S}$ of the robot centre of masses is optimally controlled, the shaking force will be minimized.

Let us consider a robot composed of $n$ links. The mass of the link $i$ is denoted as $m_{i}(i=1, \ldots, n)$ and the position of its centre of masses as $\mathbf{x}_{S i}$. Once the articular coordinates $\mathbf{q}$ or Cartesian variables $\mathbf{x}$ are known, the values of $\mathbf{x}_{S i}$ may easily be obtained using the robot kinematics relationships. As a result, the position of the robot centre of masses, defined as

$$
\mathbf{x}_{S}=\frac{1}{m_{\text {tot }}} \sum_{i=1}^{n} m_{i} \mathbf{x}_{S i}
$$

may be expressed as a function of $\mathbf{x}$ or $\mathbf{q}$. It may also be shown that, if $\operatorname{dim}\left(\mathbf{x}_{S}\right)=$ $\operatorname{dim}(\mathbf{q})(=\operatorname{dim}(\mathbf{x})$ in the case of non redundant robots), $\mathbf{x}$ and $\mathbf{q}$ may be expressed as a function of $\mathbf{x}_{S}$. We would like to mention that the case where $\operatorname{dim}\left(\mathbf{x}_{S}\right)<\operatorname{dim}(\mathbf{q})$ will not be considered in this paper. It will be the topic of our further research works.

Thus, taking into account that $\mathbf{q}=\mathbf{f}\left(\mathbf{x}_{S}\right)$ (and as a consequence $\mathbf{x}=\mathbf{g}\left(\mathbf{x}_{S}\right)$ ), the problem remains to optimally define the trajectory $\mathbf{x}_{S}(t)$. For this reason, let us consider the displacement $\mathbf{x}_{S}$ of a point $S$ in the Cartesian space. We assume that, at any moment during the displacement, the norm of the maximal admissible acceleration the point $S$ can reach is constant and denoted as $\ddot{x}_{S}^{\max }$. Taking that into consideration, the displacement law that minimize the time interval $\left(t_{0}, t_{f}\right)$ for going from position $\mathbf{x}_{\mathbf{S 0}}$ $=\mathbf{x}_{\mathbf{S}}\left(t_{0}\right)$ to position $\mathbf{x}_{\mathbf{S f}}=\mathbf{x}_{\mathbf{S}}\left(t_{f}\right)$ is the "bang-bang" law (Khalil and Dombre, 2002), given by (Fig. 1)

with

$$
\left\{\begin{array}{l}
\mathbf{x}_{S}(t)=s(t)\left(\mathbf{x}_{S \mathrm{f}}-\mathbf{x}_{S \mathbf{0}}\right)+\mathbf{x}_{S \mathbf{0}} \\
\dot{\mathbf{x}}_{S}(t)=\dot{s}(t)\left(\mathbf{x}_{S \mathrm{f}}-\mathbf{x}_{S \mathbf{0}}\right) \\
\ddot{\mathbf{x}}_{S}(t)=\ddot{s}(t)\left(\mathbf{x}_{S \mathrm{f}}-\mathbf{x}_{S \mathbf{0}}\right)
\end{array}\right.
$$

$$
\ddot{s}(t)=\frac{1}{\left\|\mathbf{x}_{S \mathbf{P}}-\mathbf{x}_{S 0}\right\|}\left\{\begin{array}{l}
\ddot{x}_{S}^{\max } \text { for } t \leq\left(t_{f}-t_{0}\right) / 2 \\
-\ddot{x}_{S}^{\max } \text { for } t \geq\left(t_{f}-t_{0}\right) / 2
\end{array}\right.
$$




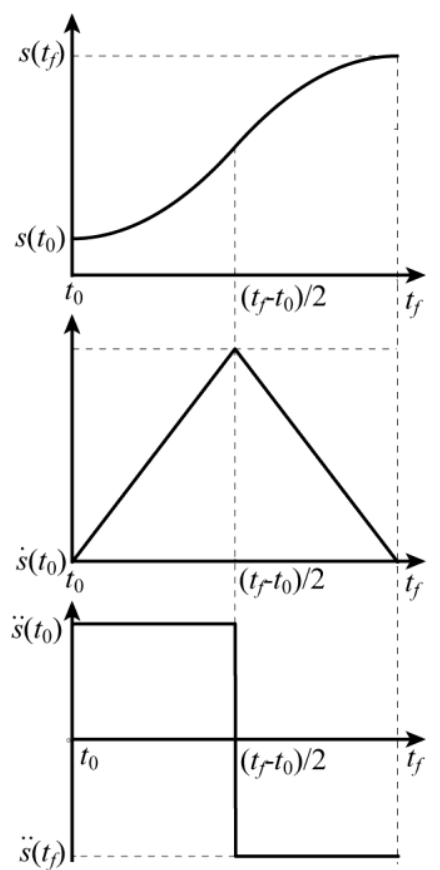

Figure 1. "Bang-bang" displacement law.

Consequently, if the time interval $\left(t_{0}, t_{f}\right)$ for the displacement between positions $\mathbf{x}_{\mathbf{S O}}$ and $\mathbf{x}_{\mathbf{S f}}$ is fixed, the "bang-bang" law is the trajectory that minimizes the value of the maximal acceleration $\ddot{x}_{S}^{\max }$ (Khalil and Dombre, 2002). Thus, in order to minimize $\left\|\ddot{\mathbf{x}}_{S}\right\|$ for a displacement during the fixed time interval $\left(t_{0}, t_{f}\right)$, the "bang-bang" law has to be applied on the displacement $\mathbf{x}_{S}$ on the robot total mass centre.

\section{Illustrative Example}

For illustration of the efficiency of the suggested solution, let us consider a planar $2 R$ serial robot (Fig. $2 \mathrm{~b}$ ) with following parameters:

- $\quad l_{O A}=0.5 \mathrm{~m}, l_{A B}=0.3 \mathrm{~m}$, where $l_{O A}$ and $l_{A B}$ are the lengths of segments $O A$ and $A B$, respectively;

- $\quad r_{1}=0.289$, where $l_{O S 1}=r_{1} l_{O A}$ and $r_{2}=0.098$, where $l_{A S 2}=r_{2} l_{A B}, l_{O S 1}$ and $l_{A S 2}$ being the lengths of segments $O S_{1}$ and $A S_{2}$, respectively.

Its mass and inertia parameters are: 
- $\quad m_{1}=24.4 \mathrm{~kg}$ and $m_{2}=8.3 \mathrm{~kg}$, where $m_{i}$ is the mass of element $i(i=1,2)$;

- $\quad m_{\text {tool }}=5 \mathrm{~kg}$, where $m_{\text {tool }}$ is the payload;

- $\quad I_{1}=1.246 \mathrm{~kg} \cdot \mathrm{m}^{2}$ and $I_{2}=0.057 \mathrm{~kg} \cdot \mathrm{m}^{2}$, where $I_{i}$ is the axial moment of inertia of element $i$.

We would like to note that the mentioned parameters correspond to the IRCCyN's robot parameters (Fig. 2), which will be used in future for experimental tests and validation of the force minimization approach developed in the present work.

In order to have the possibility to control the robot, let us express the articulated joint positions $\mathbf{q}=\left[q_{1}, q_{2}\right]^{T}$ as a function of the position $\mathbf{x}_{S}$ of the robot centre of masses. From (3), we obtain:

$$
\mathbf{x}_{S}=\left[\begin{array}{l}
x_{S} \\
y_{S}
\end{array}\right]=\frac{m_{1} r_{1} l_{O A}}{m_{t o t}}\left[\begin{array}{c}
\cos q_{1} \\
\sin q_{1}
\end{array}\right]+\frac{m_{2}}{m_{t o t}}\left(l_{O A}\left[\begin{array}{c}
\cos q_{1} \\
\sin q_{1}
\end{array}\right]+r_{2} l_{A B}\left[\begin{array}{c}
\cos \left(q_{1}+q_{2}\right) \\
\sin \left(q_{1}+q_{2}\right)
\end{array}\right]\right)
$$

This expression leads to:

$$
\left(x_{S}-l_{e q 1} \cos q_{1}\right)^{2}+\left(y_{S}-l_{e q 1} \sin q_{1}\right)^{2}-l_{e q 2}^{2}=0
$$

where $l_{e q 1}=\left(m_{1} r_{1}+m_{2}\right) l_{O A} / m_{t o t}$ and $l_{e q 2}=m_{2} r_{2} l_{A B} / m_{t o t}$.

Replacing $\cos q_{1}$ and $\sin q_{1}$ by $\left(1-t_{1}^{2}\right) /\left(1+t_{1}^{2}\right)$ and $2 t_{1} /\left(1+t_{1}^{2}\right)\left(t_{1}=\tan \left(q_{1} / 2\right)\right)$, respectively, and developing (7), we obtain:

$$
q_{1}=2 \tan ^{-1}\left(\frac{-b \pm \sqrt{b^{2}-c^{2}+a^{2}}}{c-a}\right)
$$

where

$$
a=-2 l_{e q 1} x_{S}, b=-2 l_{e q 1} y_{S} \text { and } c=x_{S}^{2}+y_{S}^{2}+l_{e q 1}^{2}-l_{e q 2}^{2} .
$$

In expression (8), the sign \pm stands for the two possible working modes of the robot (for simulations, the working mode with the "+" sign is used). Once $q_{1}$ is known, $q_{2}$ may easily be found from (6):

$$
q_{2}=\tan ^{-1}\left(\frac{y_{S}-l_{e q 1} \sin q_{1}}{x_{S}-l_{e q 1} \cos q_{1}}\right)-q_{1} .
$$

Now, let us consider a pick-and-place displacement between two positions of the end-effector $\mathbf{x}_{\mathbf{0}}=[0.3 \mathrm{~m}, 0.7 \mathrm{~m}]^{T}$ and $\mathbf{x}_{\mathbf{f}}=[0.3 \mathrm{~m}, 0.4 \mathrm{~m}]^{T}$, beginning at $t_{0}=0 \mathrm{~s}$ and finishing at $t_{f}=0.25 \mathrm{~s}$. Two cases will be simulated:

1. a fifth order polynomial law is applied on the displacement of the robot's end-effector (Eq. (2b)); 


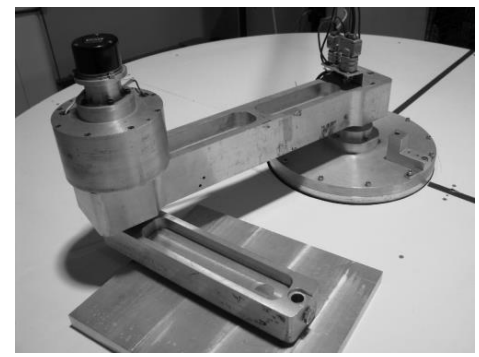

(a)

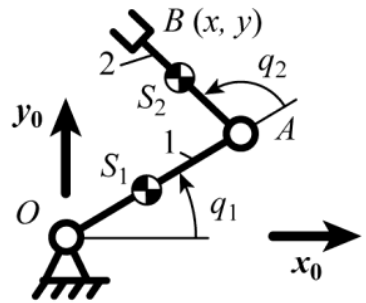

(b)

Figure 2. The $2 R$ serial robot: (a) prototype; (b) schematics.

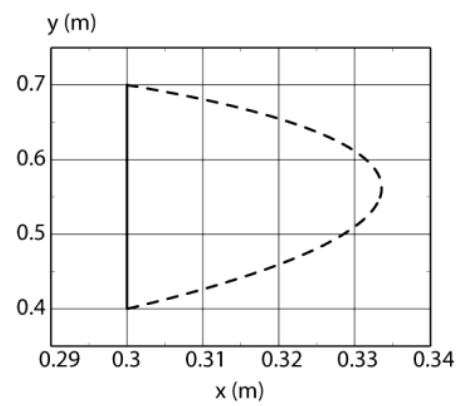

Figure 3. Displacement of the end-effector for two examined cases:

1) fifth order polynomial law applied on the displacement of the end-effector (full line) 2) "bang-bang" law applied on the displacement of the robot centre of masses (dotted line).

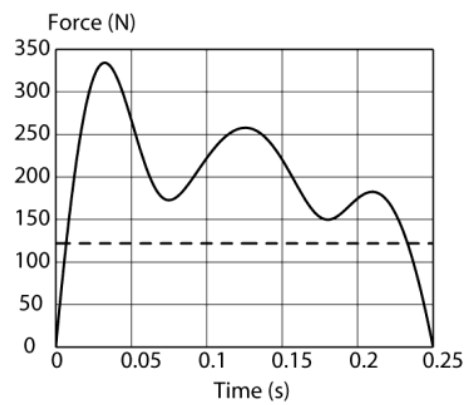

Figure 4. Shaking forces for two examined cases:

1) fifth order polynomial law applied on the displacement of the end-effector (full line)

2) "bang-bang" law applied on the displacement of the robot centre of masses (dotted line). 


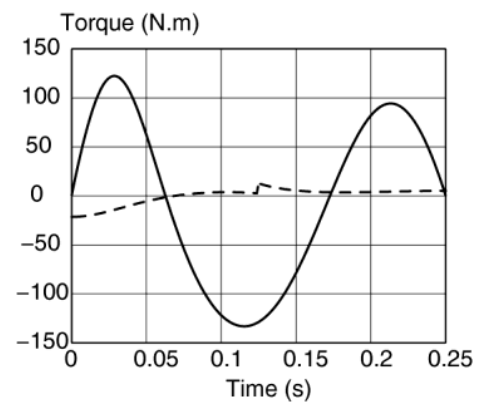

(a) actuator $q_{1}$

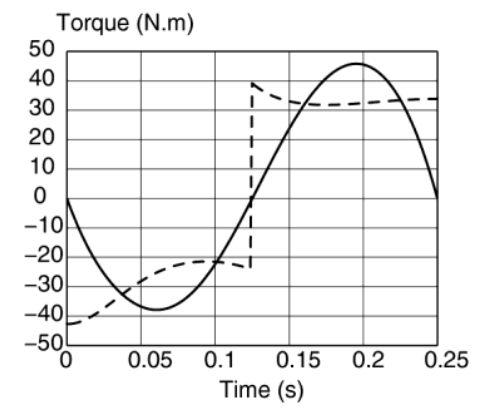

(b) actuator $q_{2}$

Figure 5. Input torques for two examined cases:

1) fifth order polynomial law applied on the displacement of the end-effector (full line)

2) "bang-bang" law applied on the displacement of the robot centre of masses (dotted line).

2. a "bang-bang" law is applied on the displacement of the robot centre of masses (Eq. (5));

The resulting displacements of the end-effector are shown in Fig. 3. These trajectory parameters are implemented into ADAMS software and we obtain the variations of shaking forces (Fig. 4). The obtained results shown that the optimal trajectory planning (case 2) allows the reduction of the shaking forces up to $63 \%$.

It should be noted that the optimal trajectory planning (case 2) has a good influence also on the input torque reduction (Fig.5). For example, in the case of the first actuator, the reduction in the input torque is $84 \%$.

\section{Conclusions}

In this paper, we have presented a new approach, based on an optimal trajectory planning, which allows the considerable reduction of the shaking forces transmitted to the robot frame. The aim of the suggested method is to optimally control the acceleration of the robot centre of masses using "bang-bang" displacement law. In other words, in the suggested approach, the robot is controlled not by applying end-effector trajectories but by planning the displacements of the total mass centre of moving links. The trajectory of the total mass centre of moving links is defined as straight line and it is parameterized with "bang-bang" displacement law. Such a control approach allows the reduction of the acceleration of the total mass centre of moving links and, consequently, the reduction in the shaking forces. It should be noted that such a solution is also very favourable for reduction of input torques. Numerical simulations carried out using ADAMS software for a planar $2 R$ serial 
manipulator have shown that reductions in the shaking force of $63 \%$ and in input torque of $84 \%$ have been achieved.

\section{References}

Arakelian, V., and Briot, S. (2008). Dynamic balancing of the SCARA robot. In Proceedings of the 17th CISM-IFToMM Symposium on Robot Design, Dynamics, and Control (Romansy 2008), Tokyo, Japan, July 5-9.

Arakelian, V, Dahan, M., and Smith, M.R. (2000). A historical review of the evolution of the theory on balancing of mechanisms. In Proceedings of the International symposium on history of machines and mechanisms. Dordrecht: Kluwer Academic Publishers. 291-300.

Arakelian, V, and Smith, M.R. (1999). Complete shaking force and shaking moment balancing of linkages. Mechanism and Machine Theory. 34(8). 1141-1153.

Arakelian, V, and Smith, M.R. (2005). Shaking force and shaking moment balancing of mechanisms: a historical review with new examples. Transactions of the ASME, Journal of Mechanical Design. 127. 334-339.

Arakelian, V. and Smith, M.R. (2008). Design of planar 3-DOF 3-RRR reactionless parallel manipulators. Mechatronics, 18, 601-606.

Berkof, R.S. (1973). Complete fore and moment balancing of inline four-bar linkages. Mechanism and Machine Theory. 8(3). 397-410.

Dresig, H., Naake, S., and Rockausen L. (1994). Vollständiger und harmonischer Ausgleich ebener Mechanismen.VDI Verlag, Düsseldorf, 73p.

Fattah, A., and Agrawal, S.K. (2006). On the design of reactionless 3-DOF planar parallel mechanisms. Mechanism and Machine Theory. 41(1). 70-82.

Gosselin, C.M., Cote, G., and Wu, Y. (2004). Synthesis and design of reactionless tree-degree-of-freedom parallel mechanisms. IEEE Transactions on Robotics and Automation. 20(2). 191-199.

Herder, J.L., and Gosselin, C.M. (2004). A counter-rotary counterweight for light-weight dynamic balancing. In Proceedings of ASME 2004 DETC/CIEC Conference, September 28 - October 2, Salt Lake City, Utah, USA. 659-667.

Khalil, W., and Dombre, E. (2002). Modeling, identification and control of robots. Hermes Sciences Europe.

Lowen, G.G., Tepper, F.R., and Berkof, R.S. (1983). Balancing of linkages - an Update. Mechanism and Machine Theory. 18(3). 213-230.

Papadopoulos, E., and Abu-Abed, A. (1994). Design and motion planning for a zero-reaction manipulator. In Proceedings of the IEEE International Conference on Robotics and Automation, San Diego, CA. 1554-1559.

Ricard, R., and Gosselin, C.M. (2000). On the design of reactionless parallel manipulators. In Proceedings of the ASME 2000 DETC/CIEC Conference, Baltimore, Maryland, September 10-13. 1-12. 